\section{REVISTA BRASILEIRA DE QUALIDADE DE VIDA}

\title{
Interpretação da sintaxe do SPSS para análise de dados do WHOQOL-100 e relação de dependência entre dados e resultados
}

\author{
Interpretation of SPSS's syntax for analysis of data of \\ WHOQOL-100 and dependency relation between data and results
}

\author{
Celso Bilynkievycz dos Santos \\ Universidade Tecnológica Federal do Paraná - UTFPR - Ponta Grossa - Brasil \\ Universidade Estadual de Ponta Grossa - UEPG - Ponta Grossa - Brasil \\ Centro Universitário Claretiano - CEUCLAR - Batatais - Brasil \\ bilynkievycz@globo.com; bilynkievycz@uepg.br
}

Bruno Pedroso

Universidade Tecnológica Federal do Paraná - UTFPR - Ponta Grossa - Brasil

brunops3@brturbo.com.br

Luciano Scandelari

Universidade Tecnológica Federal do Paraná - UTFPR - Ponta Grossa - Brasil scandelari@cits.br

Luiz Alberto Pilatti

Universidade Tecnológica Federal do Paraná - UTFPR - Ponta Grossa - Brasil lapilatti@utfpr.edu.br

\begin{abstract}
Resumo
O presente trabalho descreve as etapas de análise dos dados do WHOQOL-100 utilizando a sintaxe do software SPSS. Utilizou-se a descrição como metodologia para circunscrever a sintaxe e um modelo estruturado para representação das relações de dependência entre dados e resultados. $\mathrm{O}$ estudo resultou no detalhamento das funções de cada linha de comando da sintaxe e na identificação da relação de dependência entre questões, facetas e domínios. Conclui-se que, a partir do entendimento das relações de dependência entre os dados e resultados, a análise parcial ou completa de dados do instrumento podem ser realizadas através de outros softwares.
\end{abstract}

Palavras-chave: WHOQOL-100, sintaxe SPSS, qualidade de vida.

\begin{abstract}
This paper describes the stages of data analysis of the WHOQOL-100 using the syntax of the SPSS software. It was used description as methodology to circumscribe the syntax and a structuralized model for representation of the dependence relations between data and results. The study resulted in the detailing of the functions of each command line of the syntax and in the identification of the dependence relation between questions, facets and domains. It is concluded that, from the understanding of the dependence relations between the data and results, the partial or complete data analysis of the instrument can be carried out through others softwares.
\end{abstract}

Keywords: WHOQOL-100, SPSS's syntax, quality of life. 


\section{Introdução}

Na década de noventa ocorreu a proliferação de instrumentos para avaliação da qualidade de vida (QV). A maioria desses instrumentos foi desenvolvida nos Estados Unidos. Com efeito, cresceu, também, a ocorrência de traduções destes instrumentos (BULLINGER; ANDERSON; CELLA, 1993), embora o aproveitamento transcultural de qualquer instrumento de avaliação traduzido, sempre foi motivo de polêmica e contestação (FOX-RUSHBY; PARKER, 1995).

$\mathrm{O}$ interesse em elaborar um instrumento para avaliar a QV dentro de uma expectativa legitimamente universal fez com que a Organização Mundial da Saúde desenvolvesse um projeto internacional, o Projeto WHOQOL. O resultado destes estudos foi o WHOQOL-100, um instrumento de avaliação da QV composto por 100 itens (FOX-RUSHBY; PARKER, 1995).

O WHOQOL-100 foi elaborado com a cooperação de 15 núcleos de diferentes culturas que trabalharam simultaneamente. Anexa ao projeto foi desenvolvida uma sintaxe através do software estatístico Statistical Package for the Social Sciences (SPSS) para a realização do cálculo dos escores dos resultados obtidos em aplicações do WHOQOL-100.

A utilização de um software específico para análise dos dados e a falta de documentos descrevendo este processo ou a relação de dependência entre os dados podem constituir uma restrição do protocolo do WHOQOL-100, de forma a inibir a utilização do instrumento.

Através da pergunta norteadora da pesquisa: como analisar os dados do WHOQOL-100, utilizando a sintaxe do SPSS?, Fundamentados nos conceitos de Gil (1999) e Lakatos (2001), utilizou-se o método descritivo para descrever as etapas de análise dos dados do WHOQOL-100 e a função de cada comando da sintaxe no software SPSS. Para a definição das relações de dependência entre dados e resultados utilizou-se, segundo Sferra e Corrêa (2003), um modelo estruturado de representação.

O entendimento da sintaxe proposta pelo Grupo WHOQOL e da relação de dependência entre os atributos e resultados, possibilita a análise dos dados ou o desenvolvimento de sintaxes em outros programas de análise estatística ou gerenciadores de base de dados que calculem índices de QV através de $\mathrm{SQL}^{1}$ de atualização ou gatilhos ${ }^{2}$, melhorando o gerenciamento das informações. E, também, possibilita a análise parcial do instrumento, quando se deseja avaliar domínios ou facetas específicas.

Com base nessa perspectiva, o presente trabalho tem como objetivo principal descrever esta sintaxe, para possibilitar ao leitor o entendimento dos comandos e suas finalidades, e, como objetivo específico, identificar as relações de dependência entre as questões, facetas e domínios de QV no instrumento.

\section{WHOQOL-100}

O WHOQOL-100 é um instrumento de avaliação da QV composto por 100 questões e que analisa seis domínios: Físico, Psicológico, Nível de independência, Relações sociais, Meio Ambiente e Espiritualidade/Religião/Crenças pessoais (THE WHOQOL GROUP, 1998).

A tradução e adaptação cultural do WHOQOL-100 para o português do Brasil foi desenvolvida em 1999, sob a coordenação do pesquisador Marcelo Pio de Almeida Fleck, no Departamento de Psiquiatria e Medicina Legal da Universidade Federal do Rio Grande do Sul (FLECK et al., 1999).

\footnotetext{
${ }^{1}$ SQL - Structured Query Language ou Linguagem de Consulta Estruturada é uma linguagem de pesquisa para banco de dados (SILBERSCHATZ; KORTH; SUDARRSHAN, 1999).

${ }^{2}$ Gatilho - ou trigger é um recurso de programação presente na maioria dos sistemas de gerenciamento de banco de dados, utilizado para associar um procedimento armazenado a um evento do banco de dados (inclusão, exclusão, atualização de registro, por exemplo) de modo que o procedimento armazenado seja executado automaticamente sempre que o evento associado ocorrer (SILBERSCHATZ; KORTH; SUDARRSHAN, 1999).
} 
O instrumento proporciona uma avaliação minuciosa de 25 facetas, das quais uma corresponde às questões de QV geral e as demais correspondem a 24 aspectos de QV que estão distribuídos entre os seus domínios (Quadro 1).

Quadro 1 - Aspectos contemplados em cada domínio do WHOQOL-100

\begin{tabular}{|c|c|c|c|}
\hline \multicolumn{2}{|r|}{ Domínios } & \multicolumn{2}{|r|}{ Aspectos } \\
\hline $\mathrm{N}^{\circ}$ & Nome & $\mathrm{N}^{\circ}$ & Descrição \\
\hline \multirow{3}{*}{ I } & \multirow{3}{*}{ Físico } & 01 & Dor e desconforto \\
\hline & & 02 & Energia e fadiga \\
\hline & & 03 & Sono e repouso \\
\hline \multirow{5}{*}{ II } & \multirow{5}{*}{ Psicológico } & 04 & Sentimentos Positivos \\
\hline & & 05 & Pensar, aprender, memória e concentração \\
\hline & & 06 & Auto-estima \\
\hline & & 07 & Imagem corporal e aparência \\
\hline & & 08 & Sentimentos negativos \\
\hline \multirow{4}{*}{ III } & \multirow{4}{*}{ Nível de Independência } & 09 & Mobilidade \\
\hline & & 10 & Atividades da vida cotidiana \\
\hline & & 11 & Dependência de medicação ou de tratamentos \\
\hline & & 12 & Capacidade de trabalho \\
\hline \multirow{3}{*}{ IV } & \multirow{3}{*}{ Relações Sociais } & 13 & Relações pessoais \\
\hline & & 14 & Suporte (apoio) social \\
\hline & & 15 & Atividade sexual \\
\hline \multirow{8}{*}{$\mathrm{V}$} & \multirow{8}{*}{ Meio Ambiente } & 16 & Segurança física e proteção \\
\hline & & 17 & Ambiente no lar \\
\hline & & 18 & Recursos financeiros \\
\hline & & 19 & Cuidados de saúde e sociais: disponibilidade e qualidade \\
\hline & & 20 & Oportunidades de adquirir novas informações e habilidades \\
\hline & & 21 & Participação em, e oportunidades de recreação/ lazer \\
\hline & & 22 & Ambiente físico: poluição/ ruído/ trânsito/ clima \\
\hline & & 23 & Transporte \\
\hline VI & $\begin{array}{l}\text { Aspectos Espirituais/ Religião/ } \\
\text { Crenças pessoais }\end{array}$ & 24 & Espiritualidade/ religião/ crenças pessoais \\
\hline
\end{tabular}

Fonte: Adaptado de The WHOQOL Group (1998)

De acordo com a classificação de Gil (1999), o WHOQOL-100 coleta dados através de uma escala social de Likert, e, segundo Vasconcelos (2002), este pode ser classificado como um instrumento estruturado para surveys, porque possui redação fixa e perguntas cuja ordem, formulação e estratégia de questionamentos são invariáveis para todos os informantes, além de possibilidade de tratamento estatístico das respostas.

\section{Software SPSS}

O SPSS é um pacote estatístico para as Ciências Sociais, criado por Nie Hull e Bent. Teve sua primeira versão em 1968, e em seguida, foi desenvolvido e distribuído pela National Opinion Research Center da Universidade de Chicago, no período de 1969 e 1975.

Em 1970, com a publicação do primeiro manual do usuário do SPSS, realizada por Nie e Hull, o software obteve maior popularização entre as instituições de educação superior nos EUA.

Apesar de ser um software de análise estatística, desenvolvido para ciências sociais; é também muito utilizado em pesquisas de mercado, saúde, governo, educação e outros setores. 
A sua grande popularidade nas diversas áreas de pesquisa e a necessidade da utilização e compreensão, fizeram com que alguns pesquisadores desenvolvessem trabalhos demonstrando a utilização do software nas diversas áreas ou em suas respectivas áreas de conhecimento, como Barros (2003), Maroco (2003), Pestana e Gageiro (2005) e Pereira (2006).

O SPSS possui, uma linguagem de programação própria. Essa sintaxe facilita a repetição de análises e realização de operações não disponíveis nas caixas de diálogo do SPSS. Alguns comandos desta linguagem de programação foram adotadas pelo Grupo WHOQOL para o denvolvimento do seu protocolo de análise de dados.

\section{Análise dos dados do WHOQOL-100 utilizando a sintaxe do SPSS}

A análise de dados é de fundamental importância para o levantamento de resultados consistentes para concepção de uma conclusão da realidade em relação a determinado evento (BARROS, 2003).

Os instrumentos estruturados para surveys atendem este princípio, pois segundo Vasconcelos (2002), possuem protocolos específicos para análise dos dados. Classificado como um instrumento estruturado para surveys, o WHOQOL-100 possui uma sintaxe para análise de dados, que foi interpretada e será descrita na seção 4.2.

\subsection{Preparação dos dados e da sintaxe}

Após a coleta dos dados, devem-se gerar dois arquivos, um para armazenar os dados e outro para a sintaxe (onde são registrados os códigos de comando).

$\mathrm{O}$ arquivo onde serão tabulados os dados pode ser um arquivo de extensão "txt", do bloco de notas, ou ainda, uma tabela de um Sistema Gerenciador de Banco de Dados. Sugere-se a utilização de uma planilha do Microsoft Excel. Neste arquivo, os dados devem ser organizados em forma de uma tabela, onde, com exceção da primeira linha, cada uma das demais corresponde a um registro e cada coluna à questão analisada. A primeira coluna deve corresponder ao nome do atributo.

Para geração do arquivo da sintaxe, deve-se utilizar um arquivo do Microsoft Bloco de Notas do Microsoft Windows, que deve ser salvo com a extensão "sps". Dentro deste arquivo devem constar os comandos do Quadro 2.

Quadro 2 - Sintaxe SPSS do WHOQOL-100

\begin{tabular}{|c|c|}
\hline Linha & Comando \\
\hline 1 & $\begin{array}{l}\text { RECODE F11 F12 F13 F14 F101 F102 F103 F104 F111 F112 F113 F114 F121 F122 F123 F124 F131 F132 F133 F134 } \\
\text { F141 F142 F143 F144 F151 F152 F153 F154 F161 F162 F163 F164 F171 F172 F173 F174 F181 F182 F183 F184 F191 } \\
\text { F192 F193 F194 F21 F22 F23 F24 F201 F202 F203 F204 F211 F212 F213 F214 F221 F222 F223 F224 F231 F232 F233 } \\
\text { F234 F241 F242 F243 F244 F31 F32 F33 F34 F41 F42 F43 F44 F51 F52 F53 F54 F61 F62 F63 F64 F71 F72 F73 F74 F81 } \\
\text { F82 F83 F84 F91 F92 F93 F94 G1 G2 G3 G4 (1=1) (2=2) (3=3) (4=4) (5=5) (ELSE=SYSMIS). }\end{array}$ \\
\hline 2 & $\begin{array}{l}\text { RECODE F22 F24 F32 F34 F72 F73 F93 F94 F102 F104 F131 F154 F163 F182 F184 F222 F232 F234 (1=5) (2=4) (3=3) } \\
(4=2)(5=1) .\end{array}$ \\
\hline 3 & COMPUTE FACET1=(MEAN.3(F11,F12,F13,F14))*4. \\
\hline 4 & COMPUTE FACET2=(MEAN.3(F21,F22,F23,F24))*4. \\
\hline 5 & COMPUTE FACET3=(MEAN.3(F31,F32,F33,F34))*4. \\
\hline 6 & COMPUTE FACET4=(MEAN.3(F41,F42,F43,F44))*4. \\
\hline 7 & COMPUTE FACET5=(MEAN.3(F51,F52,F53,F54))*4. \\
\hline 8 & COMPUTE FACET6=(MEAN.3(F61,F62,F63,F64))*4. \\
\hline 9 & COMPUTE FACET7=(MEAN.3(F71,F72,F73,F74))*4. \\
\hline 10 & COMPUTE FACET8=(MEAN.3(F81,F82,F83,F84))*4. \\
\hline 11 & COMPUTE FACET9=(MEAN.3(F91,F92,F93,F94))*4. \\
\hline 12 & COMPUTE FACET10=(MEAN.3(F101,F102,F103,F104))*4. \\
\hline 13 & COMPUTE FACET11=(MEAN.3(F111,F112,F113,F114))*4. \\
\hline 14 & COMPUTE FACET12=(MEAN.3(F121,F122,F123,F124) $) * 4$. \\
\hline 15 & COMPUTE FACET13=(MEAN.3(F131,F132,F133,F134))*4. \\
\hline 16 & COMPUTE FACET14=(MEAN.3(F141,F142,F143,F144))*4. \\
\hline 17 & COMPUTE FACET15=(MEAN.3(F151,F152,F153,F154))*4. \\
\hline
\end{tabular}




\begin{tabular}{|c|c|}
\hline 18 & COMPUTE FACET16=(MEAN.3(F161,F162,F163,F164))*4. \\
\hline 19 & COMPUTE FACET17=(MEAN.3(F171,F172,F173,F174))*4. \\
\hline 20 & COMPUTE FACET18=(MEAN.3(F181,F182,F183,F184))*4. \\
\hline 21 & COMPUTE FACET19=(MEAN.3(F191,F192,F193,F194))*4. \\
\hline 22 & COMPUTE FACET20=(MEAN.3(F201,F202,F203,F204))*4. \\
\hline 23 & COMPUTE FACET21=(MEAN.3(F211,F212,F213,F214))*4. \\
\hline 24 & COMPUTE FACET22=(MEAN.3(F221,F222,F223,F224))*4. \\
\hline 25 & COMPUTE FACET23=(MEAN.3(F231,F232,F233,F234))*4. \\
\hline 26 & COMPUTE FACET24=(MEAN.3(F241,F242,F243,F244))*4. \\
\hline 27 & COMPUTE FACET25=(MEAN.3(G1,G2,G3,G4))*4. \\
\hline 28 & COMPUTE DOM1=MEAN.2((24-FACET1),FACET2,FACET3). \\
\hline 29 & $\begin{array}{l}\text { COMPUTE DOM2=MEAN.4(FACET4,FACET5,FACET6,FACET7,(24-FACET8)). } \\
\text {. }\end{array}$ \\
\hline 30 & COMPUTE DOM3=MEAN.3(FACET9,FACET10,(24-FACET11),FACET12). \\
\hline 31 & COMPUTE DOM4=MEAN.2(FACET13,FACET14,FACET15). \\
\hline 32 & 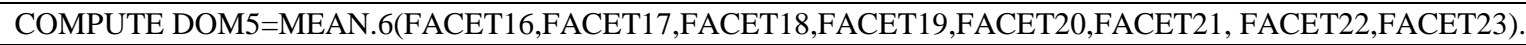 \\
\hline 33 & COMPUTE DOM6=FACET24. \\
\hline 34 & 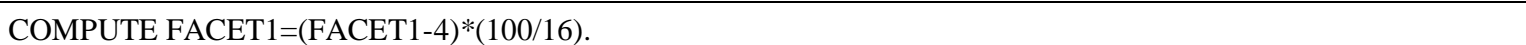 \\
\hline 35 & COMPUTE FACET2 $=($ FACET2-4 $) *(100 / 16)$. \\
\hline 36 & COMPUTE FACET3=(FACET3-4)*(100/16). \\
\hline 37 & 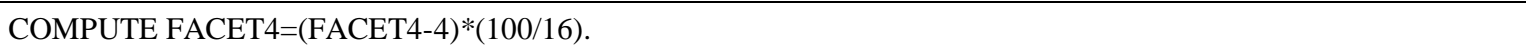 \\
\hline 38 & COMPUTE FACET5 $=($ FACET5-4 $) *(100 / 16)$. \\
\hline 39 & COMPUTE FACET6 $=($ FACET6-4 $) *(100 / 16)$. \\
\hline 40 & COMPUTE FACET7 $=($ FACET7-4 $) *(100 / 16)$ \\
\hline 41 & COMPUTE FACET $8=($ FACET $8-4) *(100 / 16)$. \\
\hline 42 & COMPUTE FACET9 $=($ FACET9-4 $) *(100 / 16)$. \\
\hline 43 & COMPUTE FACET10=(FACET10-4)*(100/16). \\
\hline 44 & COMPUTE FACET11=(FACET11-4)*(100/16). \\
\hline 45 & COMPUTE FACET12=(FACET12-4)*(100/16). \\
\hline 46 & COMPUTE FACET13=(FACET13-4)*(100/16). \\
\hline 47 & COMPUTE FACET14=(FACET14-4)*(100/16). \\
\hline 48 & COMPUTE FACET15=(FACET15-4)*(100/16). \\
\hline 49 & COMPUTE FACET16=(FACET16-4)*(100/16). \\
\hline 50 & COMPUTE FACET17=(FACET17-4)*(100/16). \\
\hline 51 & COMPUTE FACET18=(FACET18-4)*(100/16). \\
\hline 52 & COMPUTE FACET19 $=($ FACET19-4) $*(100 / 16)$. \\
\hline 53 & COMPUTE FACET20 $=($ FACET20-4 $) *(100 / 16)$. \\
\hline 54 & COMPUTE FACET21 $=($ FACET21-4)*(100/16). \\
\hline 55 & COMPUTE FACET22 $=($ FACET22-4 $) *(100 / 16)$. \\
\hline 56 & COMPUTE FACET23=(FACET23-4)*(100/16). \\
\hline 57 & COMPUTE FACET24=(FACET24-4)*(100/16). \\
\hline 58 & COMPUTE FACET25=(FACET25-4)*(100/16). \\
\hline 59 & COMPUTE DOM1=(DOM1-4)*(100/16). \\
\hline 60 & COMPUTE DOM2 $=($ DOM2-4 $) *(100 / 16)$. \\
\hline 61 & COMPUTE DOM3=(DOM3-4)*(100/16). \\
\hline 62 & COMPUTE DOM4=(DOM4-4)*(100/16). \\
\hline 63 & COMPUTE DOM5=(DOM5-4)*(100/16). \\
\hline 64 & COUNT TOTAL=F12 TO F244 (1 THRU 5). \\
\hline 65 & FILTER OFF. \\
\hline 66 & USE ALL. \\
\hline 67 & SELECT IF (TOTAL>=80). \\
\hline 68 & EXECUTE. \\
\hline
\end{tabular}

Fonte: Adaptado de The WHOQOL Group (1998)

Depois de prontos os arquivos, através do SPSS, abre-se o arquivo onde constam os dados tabulados. Caso o arquivo de origem seja uma planilha do Excel, como foi sugerido, o software SPSS, automaticamente, carregará a sua planilha com os dados do arquivo, nomeando suas colunas 
como os dados da primeira linha do arquivo. A planilha do SPSS deve ser salva com a extensão "sav".

Em seguida, pode-se abrir o arquivo da sintaxe, conforme seqüência observada na Figura 1.

Figura 1 - Seqüência de abertura do arquivo de sintaxe do SPSS

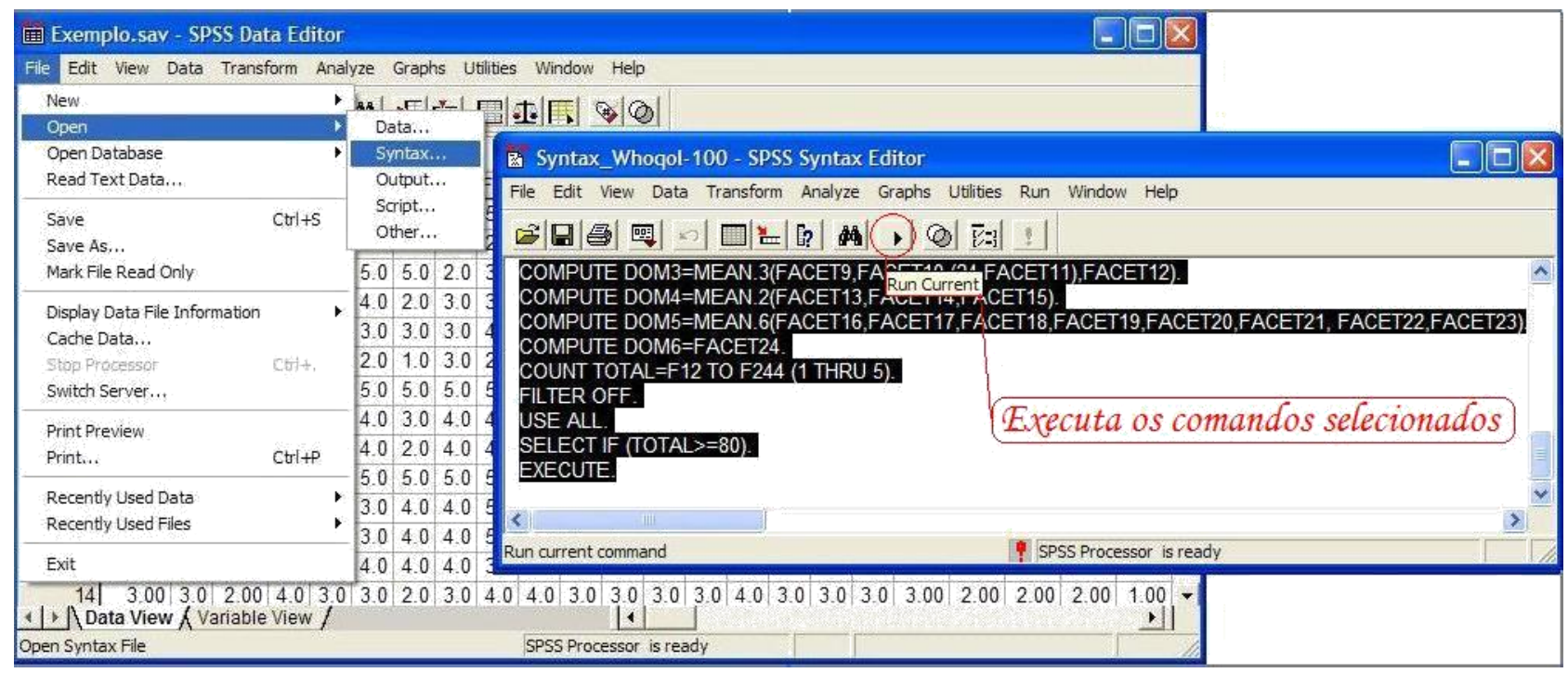

Fonte: Autoria própria (2009)

Na seqüência, devem ser selecionadas todas as linhas de comando e acionado o botão "Run Current", que executa os comandos da sintaxe.

\subsection{Sintaxe para análise dos dados do WHOQOL-100 no SPSS}

A sintaxe foi desenvolvida pelo Grupo WHOQOL, para análise de dados através do software SPSS. Mas, através do entendimento das funções de cada comando, torna-se possível o desenvolvimento de outras sintaxes, utilizando diferentes softwares.

Esta sintaxe é composta por 68 linhas (Quadro 2) de comandos que serão descritas a seguir.

A primeira linha (Quadro 2) verifica se todas as questões foram preenchidas corretamente, com as respostas 1, 2, 3, 4 ou 5. Caso contrário, a resposta ficará em branco e não será computada.

O WHOQOL-100 possui perguntas classificadas como negativas e positivas. Para o cálculo da média das facetas composta por questões com as duas categorias, deve-se considerar a leitura inversa da escala de Likert para as questões negativas. Este problema é tratado a partir da segunda linha de comando, que inverte as pontuações destas questões.

Este comando pode ser substituído, em caso da utilização de outro software, pela seguinte equação matemática, representada na Figura 2.

Figura 2 - Equação para Inversão de Escala

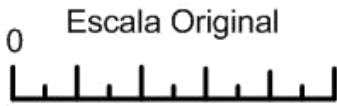

$$
\mathrm{El}=\left(\mathrm{Eo}_{\text {min }}-\mathrm{Eo}\right)+\left(\mathrm{Eo}_{\text {min }}\right) * \mathrm{~N}
$$

ou
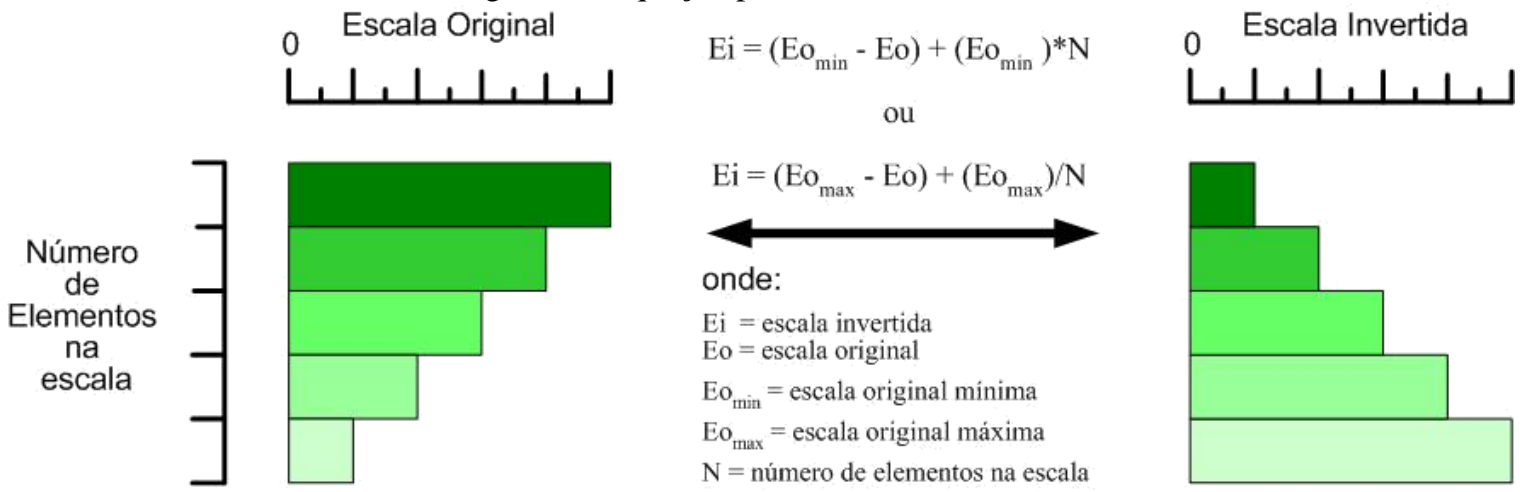

Fonte: Autoria própria (2009) 
O procedimento proposto é o mesmo adotado nos trabalhos de Rosa (2006), que desenvolveu um estudo de caso, utilizando o WHOQOL-100, sem o software SPSS para análise dos dados; Santos (2007), que desenvolveu um estudo de caso utilizando Data Mining para aquisição de conhecimento implícito nos indicadores de Qualidade de Vida, e Pedroso et al. (2008), que desenvolveu uma ferramenta no Microsoft Excel para análise do WHOQOL-100.

O pesquisador Rosa (2006), utilizou a mesma lógica matemática para desenvolvimento de uma equação de inversão de uma escala, durante a obtenção da média dos domínios. Pedroso et al. (2008) incluiu em sua ferramenta outras medidas estatísticas e aplicativos para controle da entrada de dados e geração de gráficos.

Prosseguindo a descrição dos comandos, da linha três à linha 27 (Quadro 2) dão origem a 25 novas colunas de resultados, correspondentes ao somatório de cada faceta.

A pontuação máxima de uma faceta corresponde à soma de quatro questões válidas (não nulas). Destas, duas questões avaliam um determinado aspecto e outras duas ponderam o quanto esses aspectos interferem na Qualidade de Vida.

Considerando-se a possibilidade da existência de questões não respondidas, esta sintaxe utiliza um comando que garante o mínimo de $75 \%$ das questões respondidas, descartando da análise as facetas que não atingirem esse valor. Assim, uma faceta é pontuada, somente se a mesma for avaliada (grau de satisfação) e ponderada (grau de importância). Este procedimento é garantido pelo comando da Figura 3.

Figura 3 - Linha de Comando para Calcular o escore de uma Faceta

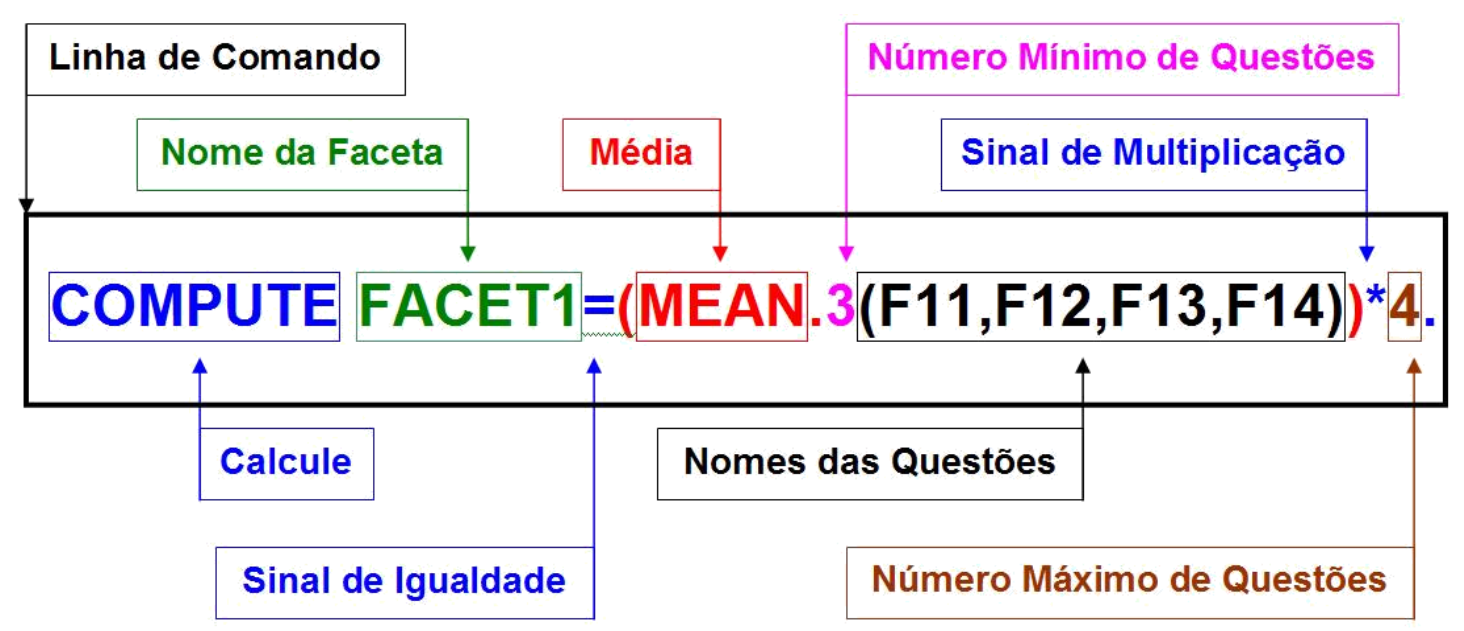

Fonte: Autoria própria (2009)

Este comando calcula a média entre o mínimo de três elementos válidos (questões respondidas), que é quadruplicada dando origem ao escore da faceta. Desta forma, para casos onde o número de questões for menor de três elementos, não se gera o valor da faceta. E quando uma faceta for composta de por apenas 3 questões válidas, a questão invalida (sem resposta) será equivalente à média entre as questões válidas.

Os comandos das linhas 28 a 33 (Quadro 2) dão origem a seis novas colunas, correspondentes à média de cada domínio. A pontuação de um domínio corresponde à média de " $n$ " facetas. Levando-se em conta, também, a possibilidade da existência de facetas com valores numéricos nulos, conseqüentes da existência de um número mínimo de questões correspondentes válidas, menor a 75\%. A sintaxe trata esses problemas nos domínios I, II III e IV com a seguinte fórmula, inserida no comando "MEAN": $(x-1)$. Onde "x" corresponde ao número de facetas do domínio.

Este comando garante que o domínio somente será calculado se possuir no máximo uma faceta não pontuada. Para o domínio 5, que contém o maior número de facetas, a sintaxe utiliza a seguinte fórmula no comando "MEAN": (x - 2), que permite o máximo de duas facetas não pontuadas. 
A linha de comando número 33 (Quadro 2) atribui a mesma equivalência da Faceta 24 ao Domínio VI, porque o domínio é composto por uma única faceta.

Da mesma forma que existem questões positivas e negativas, também, existem facetas com as duas características, que são compostas na sua totalidade por questões de uma única qualidade. Para realizar a média das facetas negativas, utiliza-se do seguinte artifício matemático: (24-FACET) incorporado ao comando para calcular os escores das facetas, invertendo, assim, a sua pontuação.

As linhas de comando de números 34 a 63 convertem os valores de uma escala contínua de 4 a 20 para uma escala centesimal, através de uma expressão equivalente a Equação 1, a seguir:

$$
E D=i E D+(E D-i E O) x \frac{\Delta E D}{\Delta E O}
$$

onde:

$$
\begin{aligned}
& \text { ED é escala desejada; } \\
& \text { EO é a escala original; } \\
& \text { i é o inicio da escala; } \\
& \text { A é a variação da escala; } e
\end{aligned}
$$

O comando da linha 64 (Quadro 2) gera uma coluna com o nome de "TOTAL", que contém a contagem de todas as questões com valores válidos.

Finalizando, as linhas de comando de 65 a 68 (Quadro 2) são responsáveis pela execução de todos os comandos, com a condição de que o número de questões válidas seja superior ou igual a de $80 \%$ do total de questões do instrumento.

De forma geral, observa-se que cada questão corresponde a um valor entre 1 e 5 , cada faceta equivale ao somatório dos escores de quatro questões que constitui, por sua vez, os domínios, correspondentes à média entre suas facetas.

Essa sintaxe pode ser enriquecida com novos comandos, para dar origem a outras medidas estatísticas descritivas, como, por exemplo, desvio padrão, entre outras.

\subsection{Relação de dependência entre dados e resultados do WHOQOL-100}

A análise de dependência visa o estudo da relação de um ou mais atributos em relação a outros atributos.

Um modelo utilizado para esse tipo de análise, descreve as dependências significativas entre variáveis. As relações de dependências podem ocorrer em dois níveis: estruturado e quantitativo. O nível estruturado de dependência é especificado, geralmente, em forma de gráfico, apresentando quais variáveis são localmente dependentes. O nível quantitativo especifica o grau de dependência, usando alguma escala numérica (SFERRA, 2003).

Geralmente são utilizados como procedimentos metodológicos para verificação de dependência as seguintes análises: discriminante, a de medidas repetidas, a de correlação canônica, a de regressão multivariada e a de variância multivariada (PADOVANI, 1995). No entanto, neste estudo, a partir da observação das linhas de comandos puderam ser definidas as relações de dependência entre as questões, faceta e domínios de QV sem a necessidade de recorrer aos métodos estatísticos.

$\mathrm{Na}$ sintaxe as questões negativas que compõem um aspecto positivo, como por exemplo, F9.3 (O quanto alguma dificuldade de locomoção lhe incomoda?), que compõem a faceta F9 (aspecto Mobilidade), sofrem uma inversão nos seus escores. O mesmo ocorre com as facetas. Portanto, segundo Crespo (2002), essa relação pode ser classificada como uma dependência direta funcional linear com proporções inversas.

Em função do conhecimento prévio das relações entre dados e resultados, observados a partir da sintaxe do SPSS, adotou-se o modelo estruturado para representação das relações de dependência, que podem ser observadas a partir da Figura 4. 
Figura 4 - Relação de Dependência entre as Questões, Facetas e Domínios de Qualidade de Vida

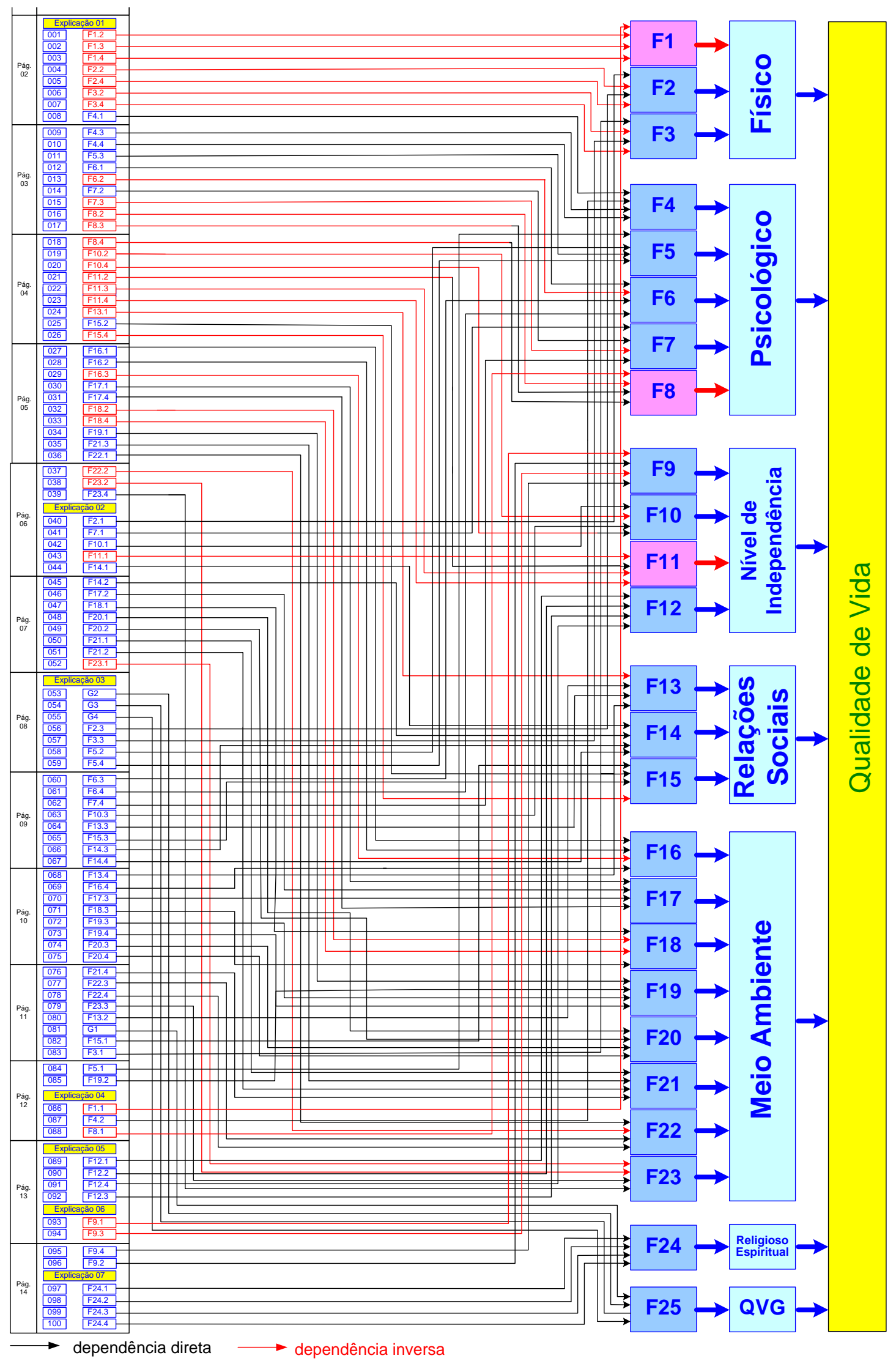

Fonte: Santos et al. (2009)

Além da representação de dependência, o diagrama da Figura 4, apresenta a distribuição das questões no instrumento, respeitando, como sugere Fleck et al. (1999), o seu layout original. 
A partir do diagrama, observa-se que a escala social é composta de 14 páginas, uma de apresentação e 13 contendo as questões, distribuídas em seis domínios relativos a diferentes aspectos de qualidade de vida. A partir do diagrama, é possível localizar na escala social, cada grupos de questões referentes a uma determinada faceta ou domínio.

O entendimento da relação de dependência entre questões/facetas/domínios e da sintaxe do SPSS poderá auxiliar na análise parcial de Qualidade de Vida, quando se deseja investigar alguns aspectos ou domínios, sem a necessidade de analisar todos dados, ou até mesmo, sem recorrer a softwares estatísticos.

\section{Conclusão}

Através da compreensão dos comandos e artifícios matemáticos utilizados na Sintaxe do SPSS para a análise dos dados do WHOQOL-100, e também do entendimento das relações de dependência entre dados (respostas na escala de Likert) e resultados (facetas e domínios), é possível desenvolver a análise dos dados em outros softwares. $\mathrm{O}$ artifício possibilita o desenvolvimento completo ou parcial do instrumento, ou ainda, o enriquecimento da sintaxe original através da adição de outros comandos e, até mesmo, dispensar a utilização de softwares estatísticos.

As facetas compostas por questões negativas e positivas, e também, os domínios compostos por facetas com esta característica, são tratados na sintaxe através de recursos matemáticos que possibilitam a inversão dos escores originais. Com efeito, é importante identificar as relações de dependência de proporções diretas ou inversas entre os atributos para evitar erros na análise dos dados.

\section{Referências}

BARROS, M. V. G. Análise de dados em atividade física e saúde: demonstrando a utilização no SPSS. Londrina: Midiograf, 2003.

BULLINGER, M.; ANDERSON, R.; CELLA, D. Developing and evaluating cross-cultural instruments from minimum requirements to optimal models. Quality of Life Research, v. 2, n. 6, p. 451-459, dec. 1993.

CRESPO, A. A. Estatística fácil. 18. ed. São Paulo: Saraiva, 2002.

FLECK, M. P. A. et al. Application of the portuguese version of the instrument for the assessment of the quality of life of the World Health Organization (WHOQOL-100). Revista de Saúde Pública, v. 33, n. 2, p. 198-205, abr. 1999.

FOX-RUSHBY, J.; PARKER, M. Culture and the measurement of health-related quality of life. Journal of European Applied Psychology, v. 45, n. 4, p. 257-263., 1995.

GIL, A. C. Métodos e técnicas de pesquisa social. 5. ed. São Paulo: Atlas, 1999.

LAKATOS, E. M.; MARCONI, M. A. Fundamentos de metodologia científica. 4. ed. São Paulo: Atlas, 2001.

MAROCO, J. Análise estatística com utilização do SPSS. Lisboa: Silabo, 2003.

PADOVANI, C. R. Estatística na metodologia da investigação científica. Botucatu: UNESP, 1995. 
PEDROSO, B. et al. Qualidade de Vida: uma ferramenta para o cálculo dos escores e estatística descritiva do WHOQOL-100. In: ENCONTRO NACIONAL DE ENGENHARIA DE PRODUÇÃO, 28., 2008, Rio de Janeiro. Anais... Rio de Janeiro: ABEPRO, 2008.

PEREIRA, A. SPSS: guia prático de utilização: análise de dados para ciências sociais e psicologia. 6. ed. Lisboa: Silabo, 2006.

PESTANA, M. H.; GAGEIRO, J. M. Descobrindo a regressão com a complementaridade do SPSS. Lisboa: Silabo, 2005.

ROSA, M. A. S. Qualidade de vida no trabalho: análise do caso de trabalhadores de uma empresa do ramo de metalurgia de Ponta Grossa - PR. 2006. 109f. Dissertação (Mestrado em Engenharia de Produção) - Programa de Pós-Graduação em Engenharia de Produção, Universidade Tecnológica Federal do Paraná, Ponta Grossa.

SANTOS, C. B. Analise dos resultados do WHOQOL-100 utilizando Data Mining. 2007. $108 f$. Dissertação (Mestrado em Engenharia de Produção) - Programa de Pós-Graduação em Engenharia de Produção, Universidade Tecnológica Federal do Paraná, Ponta Grossa.

SANTOS, C. B. et al. Aquisição de Conhecimento Implícito de Indicadores de Qualidade de Vida. Revista Brasileira de Qualidade de Vida, v. 1, n. 2, p. 33-57, jan./jun. 2009.

SFERRA, H. H.; CORRÊA, A. M. C. J. Conceitos e aplicações de Data Mining. Revista de Ciência e Tecnologia, v.11, n. 22, p. 19-34., 2003.

SILBERSCHATZ, A.; KORTH, H. F.; SUDARRSHAN, S. Sistemas de banco de dados. 3 ed. São Paulo: Makron Books, 1999.

THE WHOQOL GROUP. WHOQOL users manual. Geneva: World Health Organization, 1998.

VASCONCELOS, E. M. Complexidade e pesquisa interdisciplinar: epistemologia e metodologia operativa. Petrópolis: Vozes, 2002. 